\title{
Development of a Model for Energy Management Decisions in Refurbishment of Buildings
}

\author{
Farhad Amirifard, Fuzhan Nasiri \\ Concordia University \\ 1514 St Catherine W, Montreal, Canada \\ farhad.amirifard@concordia.ca; fuzhan.nasiri@concordia.ca
}

\section{Extended Abstract}

It had been widely accepted that the refurbishment projects are the best solutions for improving energy efficiency in aged buildings[1]. The main objective of an energy-efficient refurbishment project is to reduce energy consumption with maintaining or enhancing the indoor thermal comfort condition, as well as reducing CO2 emissions[2].The building sector consumes more than $40 \%$ of all total final energy and accounts for more than $30 \%$ of greenhouse gas emission in developed countries[3]. The stock of the existing buildings is regarded with high potential for energy performance improvement as the replacement rate of them is at a slow pace[4]. There are two possible ways of achieving energy conservation through building refurbishment; active measures or passive measures, the former deals with producing energy from sustainable and renewable technologies while the latter is concerned with optimizing energy demand. More than 400 different energy measures can be fit in these two groups which illustrate the existence of numerous alternatives that can be undertaken. Studies show that nearly $80 \%$ of energy efficiency measures are intuitively selected from these alternatives according to characteristics of the building, the location, environmental factors, etc.[5] Therefore, choosing the right combination of refurbishment actions among possible passive measures is a timely issue. This selection problem can be interpreted as a multi-objective optimization problem featured by the existence of multiple and conflicting objectives from qualitative characteristics such as occupants' behavior to quantitative criteria such as cost [6]. This paper aims at developing an integrated assessment-optimization framework to provide a decision support for prioritization and selection of building refurbishment measures with energy conservation potentials. Firstly, possible and available passive measures are investigated and identified based on six energy control principles. Secondly, an analytic network process (ANP) is utilized to calculate the relative weights of these alternative measures. These weights will be used to formulate a utility function capturing the non-monetary decision criteria. Thirdly, this "Utility" function is optimized along with the "Total refurbishment Cost" as objective functions. There are three, well-known developed methods to optimize multi-objective models with conflicting objectives including distance to ideal, compromise programming and goal programming. These methods are used to solve optimization problem by considering linear and integer programming. Finally, the proposed model is tested through a generic case study application in order to verify its applicability and usefulness. A sensitivity analysis will also be conducted to identify the most sensitive parameters of this technology selection problem to advocate strategies for uptake and promotion of passive measures in the energy conservation agenda for the building sector.

\section{References}

[1] J. Ouyang, C. Wang, H. Li, and K. Hokao, "A methodology for energy-efficient renovation of existing residential buildings in China and case study," Energy Build., vol. 43, no. 9, pp. 2203-2210, 2011.

[2] B. Güçyeter and H. M. Günaydin, "Optimization of an envelope retrofit strategy for an existing office building," Energy Build., vol. 55, no. 2012, pp. 647-659, 2012.

[3] L. Yang, H. Yan, and J. C. Lam, "Thermal comfort and building energy consumption implications - A review," Appl. Energy, vol. 115, pp. 164-173, 2014.

[4] M. Hamdy, A. Hasan, and K. Siren, "A multi-stage optimization method for cost-optimal and nearly-zero-energy building solutions in line with the EPBD-recast 2010," Energy Build., vol. 56, pp. 189-203, 2013.

[5] Y. Shao, P. Geyer, and W. Lang, "Integrating requirement analysis and multi-objective optimization for office building energy retrofit strategies," Energy Build., vol. 82, pp. 356-368, 2014. 
[6] E. Asadi, M. G. Da Silva, C. H. Antunes, and L. Dias, "Multi-objective optimization for building retrofit strategies: A model and an application," Energy Build., vol. 44, no. 1, pp. 81-87, 2012. 